\title{
Cholecalciferol/Whey Protein Isolate/EPA/DHA-based Nutritional Supplement
}

National Cancer Institute

\section{Source}

National Cancer Institute. Cholecalciferol/Whey Protein Isolate/EPA/DHA-based

Nutritional Supplement. NCI Thesaurus. Code C123916.

A gluten-free, energy-rich, non-complete nutritional supplement drink composed of juice from the concentrates of apple pear, pomegranate, purple chokeberry and passion fruit, plus fish oil derived from salmon and cod, the minerals potassium, phosphorus and iodine, whey protein isolate derived from cow's milk, tocopherols (vitamin E), and cholecalciferol (vitamin D3) with potential anti-cachexic activity. Upon oral intake of the cholecalciferol/whey protein isolate/EPA/DHA-based nutritional supplement, the protein components maintain digestive health throughout the gastrointestinal (GI) tract and reduce the risk of digestive complications. The essential omega-3 polyunsaturated fatty acids eicosapentaenoic acid (EPA) and docosahexaenoic acid (DHA) from the fish oil are incorporated into cell membranes and affect the production of pro-inflammatory mediators, which elicits an anti-inflammatory effect. Cholecalciferol plays an important role in maintaining bone mineralization and regulating blood calcium and phosphorus levels. Tocopherols neutralize free radicals, thereby protecting tissues and organs from oxidative damage. In particular, alpha-tocopherol gets incorporated into biological membranes, maintains cell membrane integrity and protects the cell against damage. This nutritional supplement may prevent both malnutrition and weight loss. 